Pacific Journal of Mathematic 


\title{
THE CARRIER SPACE OF A REFLEXIVE OPERATOR ALGEBRA
}

\author{
Alan Hopenwasser and David Larson
}

Many properties of nest algebras are actually valid for reflexive operator algebras with a commutative subspace lattice. In this paper we collect a number of such results related to the carrier space of the algebra. Included among these results are a generalization of Ringrose's criterion, a description of the partial correspondence between lattice homomorphisms of the carrier space and projections in the lattice, the construction of isometric representations of certain quotient algebras, and a direct sum decomposition of the commutant of the core modulo the intersection of the spectral ideals.

Let $\mathscr{A}=\mathrm{Alg} \mathscr{L}$, where $\mathscr{L}$ is a commutative subspace lattice and let $\mathscr{F}$ be the intersection of all the spectral ideals in $\mathscr{A}$. (See $\S 1$ for definitions.) In $\S 1$ we generalize Ringrose's criterion to the commutative subspace lattice case: $A \in \mathscr{J}$ if, and only if, for each $\varepsilon>0$ there is a finite family $\left\{E_{i}\right\}$ of mutually orthogonal intervals from $\mathscr{L}$ such that $\sum E_{i}=1$ and $\left\|E_{i} \dot{A} E_{i}\right\|<\varepsilon, i=1, \cdots, n$. We also prove that $\mathscr{I}$ is the closed linear span of commutators of the form $A L-L A$, where $A \in \mathscr{A}$ and $L \in \mathscr{L}$. In $\S 2$ we describe the partial correspondence between certain projections in $\mathscr{L}$ and certain lattice homomorphisms in the carrier space $X$. A necessary (but not sufficient) condition for an operator $A$ to be in the radical of $\mathscr{A}$ is given in $\S 3$. In $\S 4$ we exhibit isometric representations as algebras of operators acting on Hilbert space of each quotient algebra $\mathscr{A} / \mathscr{A}_{\phi}$ and of the quotient $\mathscr{A} / \mathscr{F}$. In the nest algebra case this was done by Lance in [5]. Finally, in $\S 5$ we generalize somewhat a theorem from [6] which identifies the $\mathscr{J}$-commutant of the core of $\mathscr{A}$ as the direct sum of the diagonal of $\mathscr{A}$ and $\mathscr{F}$.

1. Let $\mathscr{L}$ be a commutative subspace lattice acting on a separable Hilbert space $\mathscr{H}$, that is to say, $\mathscr{L}$ is a lattice of commuting, orthogonal projections on $\mathscr{H}$ which contains 0 and 1 and is closed in the strong operator topology. Let $\mathscr{A}=\mathrm{Alg} \mathscr{L}$, the algebra of all operators leaving invariant each projection in $\mathscr{L}$. Then $\mathscr{A}$ is a reflexive operator algebra whose lattice of invariant subspaces is just $\mathscr{L}$ [1]. Define the carrier space, $X$, of $\mathscr{L}$ to be the set of all lattice homomorphisms of $\mathscr{L}$ onto the trivial lattice $\{0,1\}$. If the carrier space is given the topology in which a net, $\phi_{\nu}$, converges to $\phi$ if, and only if, $\phi_{\nu}(L) \rightarrow \phi(L)$ for each $L \in \mathscr{L}$, then it becomes a 
compact, Hausdorff topological space.

A projection $E$ in $\mathscr{A}$ is said to be an interval if $E=L-M$ for projections $L, M \in \mathscr{L}$ with $M<L$. If $\phi \in X$, we say that $E$ is a test interval for $\phi$ if $\phi(\mathscr{L})=1$ and $\phi(M)=0$. (It is easy to check that this is well-defined.) Let $\mathscr{F}_{\phi}=\{E \mid E$ is a test interval for $\phi$ \}. $\mathscr{F}_{\phi}$ is a family of intervals which satisfies the finite intersection property and is maximal with respect to this property. Any family of intervals satisfying these conditions is called a basic family; there is a one-to-one correspondence between elements of the carrier space of $\mathscr{L}$ and basic families of intervals from $\mathscr{L}$ [3].

For each $\phi$ in $X$, define a continuous semi-norm $N_{\dot{\phi}}$ on $\mathscr{A}$ by $N_{\phi}(T)=\inf \left\{\|E T E\| \mid E \in \mathscr{F}_{\phi}\right\}$, for each $T \in \mathscr{A}$. This, in turn, permits the definition of the spectral ideal, $\mathscr{A}_{\phi}$, associated with $\phi: \mathscr{A}_{\phi}=$ $\left\{T \in \mathscr{A} \mid N_{\phi}(T)=0\right\}$. The spectral ideals are closed two-sided ideals in $\mathscr{A}$, as is the intersection, $\mathscr{F}$, of all the spectral ideals. Proofs of these facts can be found in [3], as well as the fact that $\mathscr{F}$ is contained in the radical, $\mathscr{R}$, of the algebra $\mathscr{A}$. It is known that $\mathscr{I}=\mathscr{R}$ if $\mathscr{A}$ is a nest algebra [7] and in a number of other cases, and we conjecture that equality always holds. Should it occur that $\mathscr{F}$ need not equal $\mathscr{R}$, it now appears clear that the role played by $\mathscr{F}$ in the structure of $\mathscr{A}$ is at least as important as the role played by the radical. As evidence in favor of the conjecture, we prove below that the Ringrose criterion for membership in the radical of a nest algebra ([7], Theorem 5.4) is a criterion for membership in $\mathscr{F}$ in the general case.

Each semi-norm $N_{\phi}$ on $\mathscr{A}$ can be identified with the quotient norm on $\mathscr{A} / \mathscr{A}_{\phi}$. Denote this quotient algebra by $\mathscr{D}_{\phi}$ and the canonical quotient map by $q_{\phi}$. Later, in $\S 4$, we shall exhibit an isometric representation of $\mathscr{D}_{\phi}$ as an algebra of operators acting on Hilbert space.

Proposition 1. For each $T \in \mathscr{A}, N_{\phi}(T)=\left\|q_{\dot{\phi}}(T)\right\|$.

Proof. Let $T \in \mathscr{C}$. If $E$ is a test interval for $\phi$, then $E T E-$ $T \in \mathscr{H}_{\phi}$. Hence $\left\|q_{\phi}(T)\right\| \leqq\|T+(E T E-T)\|=\|E T E\|$. Thus, $\left\|q_{\phi}(T)\right\| \leqq \inf \left\{\|E T E\| \mid E \in \mathscr{F}_{\phi}\right\}=N_{\phi}(T)$. For the opposite inequality, let $S \in \mathscr{A}_{\phi}$. Let $\varepsilon$ be an arbitrary positive number and choose a test interval $E$ for $\phi$ such that $\|E S E\|<\varepsilon$. Then

$$
\begin{aligned}
\|T+S\| & \geqq\|E(T+S) E\|=\|E T E+E S E\| \\
& \geqq\|E T E\|-\|E S E\| \\
& \geqq N_{\phi}(T)-\varepsilon .
\end{aligned}
$$

Since $\varepsilon$ is arbitrary, $\|T+S\| \geqq N_{\phi}(T)$ for all $S \in \mathscr{A}_{\phi}$. Hence $\left\|q_{\phi}(T)\right\| \geqq$ 
$N_{\phi}(T)$.

A similar result holds for the quotient algebra $\mathscr{A} / \mathscr{A}$, where $\mathscr{J}=\bigcap_{\phi \in X} \mathscr{A}_{\phi}$. If $\mathscr{D}=\mathscr{A} / \mathscr{J}$ and if $q: \mathscr{A} \rightarrow \mathscr{D}$ is the canonical quotient map, then $\sup N_{\phi}(\cdot)=\|q(\cdot)\|$. Further, for each $T \in \mathscr{A}$, the supremum is attained. In the case of a nest algebra this is just Lemma 1.6 of [6] expressed in terms of the carrier space of $\mathscr{L}$. The ingredients needed for this result also yield the validity of the Ringrose criterion as a description of the elements of $\mathscr{F}$; a consequence of this criterion is the identification of $\mathscr{J}$ as the closed linear span of all commutators $A L-L A$, with $A \in \mathscr{A}$ and $L \in \mathscr{L}$. The first preliminary result which we need is the assertion that each such commutator lies in $\mathscr{F}$.

Lemma 2. If $A \in \mathscr{A}$ and $L \in \mathscr{L}$ then $A L-L A \in \mathscr{J}$.

Proof. Let $\phi \in X$. If $\phi(L)=1$ then $L \in \mathscr{F}_{\phi}$ and $N_{\phi}(A L-L A) \leqq$ $\|L(A L-L A) L\|=0$. If $\phi(L)=0$ then $1-L \in \mathscr{F}_{\phi}$ and $N_{\phi}(A L-L A) \leqq$ $\|(1-L)(A L-L A)(1-L)\|=0$. Thus, $A L-L A \in \mathscr{A}_{\phi}$, for all $\phi \in x$; i.e., $A L-L A \in \mathscr{J}$.

Lemma 3. If $\phi \in X$ and $A \in \mathscr{A}$ then $N_{\phi}(A) \leqq\|q(A)\|$.

Proof. Since $\mathscr{J} \subseteq \mathscr{A}_{\phi}$, we have $\left\|q_{\phi}(A)\right\| \leqq\|q(A)\|$, and the lemma follows from Proposition 1.

Lemma 4. Let $A \in \mathscr{A}$. Then there exists an element $\phi \in X$ such that $N_{\psi}(A) \leqq N_{\phi}(A)$, for all $\psi \in X$.

Proof. In the remark on $p .379$ of [3] it is shown that the mapping $\psi \rightarrow N_{\psi}(A)$ of $X$ into $\boldsymbol{R}$ is upper semi-continuous. Since $X$ is a compact Hausdorff space, this mapping achieves its supremum at some point $\phi$ in $X$.

Definition. A projection in $\mathscr{A}$ is said to be simple if it is 0 or it is a finite sum of intervals from $\mathscr{L}$.

Proposition 5. The set of simple projections is a complemented lattice.

Proof. We must show that if $E$ and $F$ are simple, then $E \wedge F=$ $E F, E^{\perp}=I-E$, and $E \vee F=E+F-E F$ are simple.

First suppose that $E$ and $F$ are intervals from $\mathscr{L}$, say $E=L-M$ and $F=N-P$, where $L, M, N, P \in \mathscr{L}$ and $M \leqq L$ and $P \leqq N$. Then 


$$
E \wedge F=L \wedge N-((L \wedge P) \vee(N \wedge M)) \text {. }
$$

Since $\mathscr{L}$ is a lattice, $L \wedge N$ and $(L \wedge P) \vee(N \wedge M)$ both lie in $\mathscr{L}$; further $(L \wedge P) \vee(N \wedge M) \leqq L \wedge N$, so $E \wedge F$ is an interval. Now suppose that $E=\bigvee_{i=1}^{n} E_{i}$ and $F=\bigvee_{j=1}^{m} F_{j}$, where $\left\{E_{i}\right\}$ and $\left\{F_{j}\right\}$ are each families of mutually orthogonal intervals from $\mathscr{L}$. Then $E \wedge F=$ $\mathrm{V}_{i, j}\left(E_{i} \wedge F_{j}\right)$ and $\left\{E_{i} \wedge F_{j}\right\}$ is a family of mutually orthogonal intervals from $\mathscr{L}$; thus $E \wedge F$ is simple.

Next observe that if $E$ is an interval then $E^{\perp}$ is the sum of two orthogonal intervals and hence is simple. If $E$ is merely simple, then $E=\bigvee_{i=1}^{n} E_{i}$, where $\left\{E_{i}\right\}$ is a mutually orthogonal family of intervals from $\mathscr{L}$. Since $E^{\perp}=\bigwedge_{i=1}^{n} E_{i}^{\perp}$ and each $E_{i}^{\perp}$ is simple, the paragraph above implies that $E^{\perp}$ is simple.

It remains only to show that if $E$ and $F$ are simple, then $E \vee F$ is simple. When $E$ and $F$ are orthogonal, this is obvious; for the general case use the facts that $F \wedge E^{\perp}$ is simple and $E \vee F=E \vee$ $\left(F \wedge E^{\perp}\right)$.

For the following lemma, and for $\S 2$, recall that a subset $\mathscr{K}_{1} \subseteq \mathscr{L}$ is said to be an ideal if it satisfies the two properties:

(a) $K_{1}, K_{2} \in \mathscr{K}_{1}, \Rightarrow K_{1} \vee K_{2} \in \mathscr{K}_{1}$

(b) $K \in \mathscr{K}_{1}, L \in \mathscr{L}, L \leqq K \Rightarrow L \in \mathscr{K}_{1}$.

Similarly, a subset $\mathscr{K}_{2}$ is said to be a co-ideal if it satisfies the dual properties:

(a') $K_{1}, K_{2} \in \mathscr{K}_{2} \Rightarrow K_{1} \wedge K_{2} \in \mathscr{K}_{2}$

(b') $K \in \mathscr{K}_{2}, L \in \mathscr{L}, K \leqq L \Rightarrow L \in \mathscr{K}_{2}$.

An ideal is prime if its complement is a co-ideal.

Lemma 6. Every interval from $\mathscr{L}$ is a test interval for some lattice homomorphism in $X$.

Proof. Let $E$ be an interval from $\mathscr{L}$. Then there exist projections $L$ and $M$ in $\mathscr{L}$ with $M<L$ such that $E=L-M$. Let $\mathscr{J}_{0}=$ $\{R \in \mathscr{L} \mid R \geqq L\}$ and $\mathscr{K}_{0}=\{S \in \mathscr{L} \mid S \leqq M\}$. It is routine to check that $\mathscr{K}_{0}$ is an ideal in $\mathscr{L}$ and that $\mathscr{J}_{0}$ is a co-ideal; it is obvious that $\mathscr{K}_{0}$ and $\mathscr{J}_{0}$ are disjoint. By a result of Stone, there exists an ideal $\mathscr{K} \supseteqq \mathscr{K}_{0}$ and a co-ideal $\mathscr{J} \supseteqq \mathscr{J}_{0}$ such that $\mathscr{K} \cap \mathscr{J}=\varnothing$ and $\mathscr{K} \cup \mathscr{J}=\mathscr{L}$. (See [4], page 80.) Since $\mathscr{K}$ is a prime ideal, there is a lattice homomorphism $\dot{\phi} \in X$ such that $\mathscr{K}=\operatorname{ker} \phi([2]$, p. 28). Since $\phi(L)=1$ and $\phi(M)=0, E$ is a test interval for $\phi$.

LEMMA 7. Let $E$ be an interval from $\mathscr{L}$ and let $U=\{\phi \in X \mid E$ is a test interval for $\phi\}$. Then $U$ is a nonempty open and closed subset of $X$. 
Proof. The preceding proposition says that $U$ is nonempty. To see that $U$ is closed, suppose $\phi_{\nu} \in U$ and $\phi_{\nu} \rightarrow \phi$ in $X$. If $E=L-M$, with $L, M \in \mathscr{L}, L<M$, then $\phi_{\nu}(L)=1$ and $\phi_{\nu}(M)=0$ for all $\nu$; hence $\phi(L)=1$ and $\phi(M)=0$. Thus $\phi \in U$ and $U$ is closed. To see that $U$ is open, let $\phi_{\nu} \in X-U$ and $\phi_{\nu} \rightarrow \phi$. Since $\phi_{\nu}$ is convergent, the two nets $\phi_{\nu}(L)$ and $\phi_{\nu}(M)$ are each eventually constant. For all $\nu, \phi_{\nu}(L)=\phi_{\nu}(M)$, since $E$ is not a test interval for $\phi_{\nu}$; consequently $\phi(L)=\phi(M)$ and $\phi \in X-U$. Thus $X-U$ is closed and $U$ is open.

Lemma 8. If $E$ is a simple projection then, for any $A \in \mathscr{A}$, $E A E^{\perp}$ and $E^{\perp} A E$ lie in $\mathscr{F}$.

Proof. By Lemma 2, every projection in $\mathscr{L}$ commutes with every member of $\mathscr{A}$ modulo $\mathscr{F}$, hence finite linear combinations of such projections (and, in particular, simple projections) have this property. Therefore $E A E^{\perp}=E(E A)-(E A) E \in \mathscr{J}$ and $E^{\perp} A E=$ $(A E) E-E(A E) \in \mathscr{J}$.

Proposition 9. Let $q$ be the canonical quotient map of $\mathscr{A}$ onto $\mathscr{D}=\mathscr{A} / \mathscr{F}_{0}$ For each $A \in \mathscr{A}, \sup _{\phi \in X} N_{\phi}(A)=\|q(A)\|$. Further, the supremum is attained by some lattice homomorphism (which depends upon A).

Proof. Lemma 3 asserts that $\sup N_{\phi}(A) \leqq\|q(A)\|$, while Lemma 4 asserts that the supremum is attained. Suppose that $A$ is an element of $\mathscr{A}$ for which $\alpha=\sup N_{\phi}(A)<\|q(A)\|$. Let $\beta$ be such that $\alpha<\beta<\|q(A)\|$. For each $\phi$ in $X$ choose a test interval $E_{\phi}$ such that $\left\|E_{\phi} A E_{\phi}\right\|<\beta$. (This is possible since inf $\left\{\|E A E\| \mid E \in \mathscr{F}_{\phi}\right\}=$ $N_{\phi}(A)<\beta$.) Let $U_{\phi}=\left\{\psi \in X \mid E_{\phi} \in \mathscr{F}_{\psi}\right\}$. Since $\phi \in U_{\phi}$ for each $\phi$, the family $\left\{U_{\phi}\right\}$ is an open cover for $X$. But $X$ is compact, so there exist finitely many lattice homomorphisms $\phi_{1}, \cdots, \phi_{n} \in X$ such that $U_{\dot{\phi}_{1}}, \cdots, U_{\phi_{n}}$ cover $X$.

Observe that $\bigvee_{i=1}^{n} E_{\phi_{i}}=1$. Indeed, by Proposition 5, the complement of $\mathrm{V}_{i=1}^{n} E_{\phi_{i}}$ is simple. If the complement is not 0 , it must contain an interval projection $F$. Let $\phi_{0}$ be a lattice homomorphism for which $F$ is a test interval. Since $F \wedge E_{\phi_{i}}=0$ for $i=1, \cdots, n$, none of the $E_{\phi_{i}}$ are test intervals for $\phi_{0}$. But this says that $\phi_{0} \notin$ $\bigcup_{i=1}^{n} U_{\phi_{i}}=X$, a contradiction.

Now define $F_{1}=E_{\phi_{1}}$ and $F_{k}=E_{\phi_{k}} \wedge\left(F_{1} \vee \cdots \vee F_{k-1}\right)^{\perp}$, for $k=$ $2, \cdots, n$. Then $F_{1}, \cdots, F_{n}$ are mutually orthogonal, each $F_{i}$ is a sub-projection of $E_{\phi_{i}}$, and $\sum_{i=1}^{n} F_{i}=\bigvee_{i=1}^{n} F_{i}=\bigvee_{i=1}^{n} E_{\phi_{i}}=1$. It follows that $\left\|q\left(F_{i} A F_{i}\right)\right\| \leqq\left\|q\left(E_{\phi_{i}} A E_{\phi_{i}}\right)\right\| \leqq\left\|E_{\phi_{i}} A E_{\phi_{i}}\right\|<\beta$, for each $i$; by [6], Lemma 1.1, $\left\|q\left(\sum_{i=1}^{n} F_{i} A F_{i}\right)\right\|=\max _{i=1, \ldots, n}\left\|q\left(F_{i} A F_{i}\right)\right\|<\beta<\|q(A)\|$. Since $A=\sum_{i, j} F_{i} A F_{j}$, we will obtain a contradiction if we show 
that $\left\|q\left(\sum_{i=1}^{n} F_{i} A F_{i}\right)\right\|=\left\|q\left(\sum_{i, j=1}^{n} F_{i} A F_{j}\right)\right\|$. But this equality follows from Lemma 8 , the fact that each $F_{i}$ is simple, and the observation that $F_{2} \perp F_{j}$ if $i \neq j$. This proves the proposition.

If $\mathscr{A}$ is a nest algebra, the following theorem is precisely the Ringrose criterion ([7], Theorem 5.4).

THEOREm 10. Let $A \in \mathscr{A}$. Then $A \in \mathscr{I}$ if, and only if, given $\varepsilon>0$ othere exists a finite family, $\left\{E_{i}\right\}_{i=1, \ldots, k}$, of mutually orthogonal intervals from $\mathscr{L}$ such that $\sum_{i=1}^{k} E_{i}=1$ and $\left\|E_{i} A E_{i}\right\|<\varepsilon$, for all $i$.

Proof. It is clear that any operator in $\mathscr{A}$ which satisfies this condition must lie in each $\mathscr{A} \phi$, and hence in $\mathscr{F}$. For the converse, suppose $A \in \mathscr{F}$. Let $\varepsilon>0$. For each $\phi \in X$ there is a test interval $E_{\phi}$ in $\mathscr{F}_{\phi}$ such that $\left\|E_{\phi} A E_{\phi}\right\|<\varepsilon$. Let $U_{\phi}=\left\{\psi \in X \mid E_{\phi} \in \mathscr{F}_{\psi}\right\}$. The family $\left\{U_{\phi}\right\}$ is an open cover for $X$; let $U_{\phi_{1}}, \cdots, U_{\phi_{n}}$ be a finite subcover. Just as in the proof of Proposition 9, $\bigvee_{i=1}^{n} E_{\phi_{i}}=1$. Since the simple projections form a complemented lattice, there is a finite sequence, $\left\{E_{i}\right\}_{i=1, \ldots, k}$, of mutually orthogonal intervals such that $1=$ $\sum_{i=1}^{k} E_{\imath}$ and, for each $i$ and $j$, either $E_{i} \leqq E_{\phi_{j}}$ or $E_{i} \perp E_{\phi_{j}}$. Consequently, $\left\|E_{i} T E_{i}\right\|<\varepsilon$ for all $i$ and the theorem is proven.

If $A \in \mathscr{A}$ and $L \in \mathscr{L}$ then $L A-A L=L A L^{\perp}$; from this it is clear that the linear span of such commutators is an ideal in $\mathscr{A}$. As a corollory of Theorem 10 we can identify $\mathscr{F}$ as the closure of this ideal.

THEOREM 11. The closure of the linear span of the set of commutators of the form $L A-A L$, with $A \in \mathscr{C}$ and $L \in \mathscr{L}$, is the ideal $\mathscr{F}$.

Proof. Lemma 2 shows that the closure is contained in $\mathscr{F}$. Let $\mathscr{F}_{0}$ denote the linear span of commutators $L A-A L, A \in \mathscr{A}, L \in \mathscr{L}$. From Theorem 10 it is sufficient to prove that if $\left\{E_{i}\right\}_{i=1, \cdots, n}$ is a family of mutually orthogonal intervals such that $\sum_{i=1}^{n} E_{i}=1$ and if $A \in \mathscr{I}$ then $A-\sum_{i=1}^{n} E_{i} A E_{i} \in \mathscr{I}_{0}$. The argument in Lemma 8 shows that, for each $i, E_{i} A-E_{i} A E_{i}=E_{i} A E_{i}^{\perp}$ is in $\mathscr{F}_{0}$. Hence $A-\sum_{i=1}^{n} E_{i} A E_{i}=\sum_{i=1}^{n} E_{i} A-\sum_{i=1}^{n} E_{i} A E_{i}=\sum_{i=1}^{n}\left(E_{i} A-E_{i} A E_{i}\right) \in \mathscr{J}_{0}$.

2. Although the carrier space appears in the literature on nest algebras $[5,7]$, it does so in disguise. The reason for the disguise is that the carrier space can be parameterized in a natural way by the projections in the nest $\mathscr{L}$. Each projection $L \neq 0,1$ corresponds to two lattice homomorphisms: define $\phi_{L}^{+}$by the requirement that it 
map $L$ and each subprojection of $L$ to 0 and all other projections to 1 ; define $\phi_{L}^{-}$by requiring that it map each proper subprojection of $L$ to 0 and all other projections to 1 . The projections 0 and 1 each correspond to a single lattice homomorphism, $\dot{\phi}_{0}^{+}$and $\phi_{1}^{-}$, respectively. The lattice homomorphisms so obtained are all distinct except when $L$ is an immediate predecessor to $M$, in which case $\phi_{L}^{+}=\phi_{M}^{-}$. It is easy to see that each element of the carrier space arises in this fashion. Given $L \neq 1$, the family of all intervals of the form $M-L$, with $M>L$, is a "cofinal" subfamily of the basic family for $\phi_{L}^{+}$and is used in place of the basic family. A similar remark applies for homomorphisms of the form $\phi_{L}^{-}$.

In the general case, in which $\mathscr{L}$ is a commutative subspace lattice, this correspondence partially breaks down. Not every projection in $\mathscr{L}$ gives rise to a lattice homomorphism and not every homomorphism is associated with a projection in $\mathscr{L}$. In this section we describe that portion of the correspondence which remains valid.

Let $L \in \mathscr{L}$, with $L \neq 1$. The set $[0, L]=\{M \in \mathscr{L} \mid M \leqq L\}$ is an ideal in the lattice $\mathscr{L}$; its complement, $[0, L]^{c}=\{M \in \mathscr{L} \mid M \nsubseteq L\}$ need not be a co-ideal. (Although it is true that if $N \in[0, L]^{c}$ and $M \geqq N$ then $M \in[0, L]^{c}$, it is not necessarily the case that $N_{1}, N_{2} \in$ $[0, L]^{c}$ implies $N_{1} \wedge N_{2} \in[0, L]^{c}$.) The mapping $\phi: \mathscr{L} \rightarrow\{0,1\}$ which maps each projection in $[0, L]$ to 0 and each projection in $[0, L]^{c}$ to 1 is a lattice homomorphism if, and only if, $[0, L]^{c}$ is a co-ideal. Similarly, if $L \neq 0$, the set $[L, 1]=\{M \mid M \geqq L\}$ is automatically a co-ideal while its complement, $[L, 1]^{c}=\{M \mid M \nsupseteq L\}$, need not be an ideal. The mapping $\phi: \mathscr{L} \rightarrow\{0,1\}$ which takes the value 1 on $[L, 1]$ and 0 on $[L, 1]^{c}$ is a lattice homomorphism if, and only if, $[L, 1]^{c}$ is an ideal.

An interval $E$ in a nest algebra can be written in the form $E=L-M$ for a unique choice of $L, M \in \mathscr{L}$ with $M<L$. While this is not necessarily true in the general commutative case, it is possible to define upper and lower endpoints for $E$.

Definition. Let $E$ be an interval from $\mathscr{L}$. Define the upper endpoint of $E$ to be the projection $P=\Lambda\{L \in \mathscr{L} \mid E \leqq L\}$ and define the lower endpoint of $E$ to be the projection $Q=\mathrm{V}\{L \in \mathscr{L} \mid E \perp L\}$.

In general it is not necessarily true that $Q \leqq P$. However, we do have $E=P \vee Q-Q=P-P \wedge Q=P(I-Q)$.

We can now single out a class of projections in $\mathscr{L}$, the fundamental projections, which give rise to lattice homomorphisms in $X$.

Definition. A projection $L \neq 1$ in $\mathscr{L}$ is said to be upper fun- 
damental if $L$ is the lower endpoint of every interval of the form $M-L$, with $M \in \mathscr{L}, M>L$. A projection $L \neq 0$ is said to be lower fundamental if $L$ is the upper endpoint of every interval of the form $L-M$, with $M \in \mathscr{L}, M<L$.

Lemma 12. (a) $L$ is upper fundamental $\Leftrightarrow[0, L]^{c}$ is a co-ideal. (b) $L$ is lower fundamental $\Leftrightarrow[L, 1]^{\circ}$ is an ideal.

Proof. (a) Assume $L$ is upper fundamental. Suppose $[0, L]^{\circ}$ is not a co-ideal: then there exist $M_{1}, M_{2} \in[0, L]^{c}$ such that $M_{1} \wedge M_{2} \leqq L$. Since $M_{1} \not \leq L, L \vee M_{1}-L \vee\left(M_{1} \wedge M_{2}\right)=L \vee M_{1}-L$ is a nonzero interval. Further, since $M_{1} \wedge M_{2} \leqq L, M_{2} \perp\left(L \vee M_{1}-L\right)$. Since $L$ is the lower endpoint of $L \vee M_{1}-L, M_{2} \leqq L$, a contradiction. Thus $[0, L]^{c}$ is a co-ideal.

Now suppose $[0, L]^{c}$ is a co-ideal. Let $M \in \mathscr{L}$ with $M>L$. To prove that $L$ is lower fundamental, we must show that if $N \perp(M-L)$ then $N \leqq L$. But $N \perp(M-L)$ implies that $N \wedge M=L \wedge N \leqq L$, i.e., $N \wedge M \notin[0, L]^{c}$. Since $M \in[0, L]^{c}$ and $[0, L]^{c}$ is a co-ideal, we cannot have $N \in[0, L]^{c}$. Thus $N \leqq L$ as desired.

(b) The proof is just the dual of the argument in (a).

For each $L \neq 1$, define a mapping $\phi_{L}^{+}: \mathscr{L} \rightarrow\{0,1\}$ by requiring $\phi_{L}^{+}$ to take the value 0 on $[0, L]$ and the value 1 on $[0, L]^{c}$. For each $L \neq 0$, define $\phi_{L}^{-}: \mathscr{L} \rightarrow\{0,1\}$ by requiring $\phi_{\bar{L}}^{-}$to take the value 1 on $[L, 1]$ and the value 0 on $[L, 1]^{c}$. We then have the following corollary:

CoRollary 13. (a) $\phi_{L}^{+} \in X \Leftrightarrow L$ is upper fundamental.

(b) $\phi_{\bar{L}} \in X \Leftrightarrow L$ is lower fundamental.

In order to describe which lattice homomorphisms are associated with fundamental projections, we recapitulate the classification of points in $X$ in [3]. Fix an element $\phi \in X$ and define

$$
\begin{aligned}
& K_{0}(\phi)=\bigvee\{L \in \mathscr{L} \mid \phi(L)=0\}=\mathrm{V} \operatorname{ker} \phi \\
& K_{1}(\phi)=\Lambda\{L \in \mathscr{L} \mid \phi(L)=1\}=\Lambda \text { coker } \phi .
\end{aligned}
$$

When $\phi$ is understood, we write $K_{0}$ and $K_{1}$ in place of $K_{0}(\phi)$ and $K_{1}(\phi)$.

DeFinition. If $\phi\left(K_{0}\right)=0$, we say that $\phi$ is upper adjacent to $K_{0}$. If $\phi\left(K_{1}\right)=1$, we say that $\phi$ is lower adjacent to $K_{1}$.

It is easy to check that if $\phi$ is upper adjacent to $K_{0}$, then $\left[0, K_{0}\right]^{\circ}$ is a co-ideal and $K_{0}$ is upper fundamental. Similarly, if $\phi$ is lower adjacent to $K_{1}$, then $\left[K_{1}, 1\right]^{\circ}$ is an ideal and $K_{1}$ is lower fundamental. 
In general the four possibilities $K_{0}=K_{1}, K_{0}<K_{1}, K_{0}>K_{1}$, and $K_{0}$ and $K_{1}$ not comparable will each occur. The four further possibilities arising from the fact that each of $K_{0}, K_{1}$ may be in either $\operatorname{ker} \phi$ or in coker $\phi$ also occur. Of the 16 a priori combinations, precisely 7 can occur. (See [3] for proofs and examples.) These 7 differenf types of lattice homomorphisms are described as follows:

Definition. $\dot{\phi}$ is said to be atomic if $\phi\left(K_{0}\right)=0$ and $\phi\left(K_{1}\right)=1$.

If $\phi$ is atomic we may have either $K_{0}<K_{1}$ or $K_{0}$ not comparable with $K_{1}$; if either of these two conditions hold, then $\phi$ must be atomic. If $\phi$ is atomic then $\phi$ is both upper adjacent to $K_{0}$ and lower adjacent to $K_{1}$. The atomic lattice homomorphisms are the only ones which arise from two distinct fundamental projections. When $\phi$ is atomic, $K_{1}-K_{0} \wedge K_{1}$ is a minimal projection in the basic family $\mathscr{F}_{\phi}$.

Definition. $\phi$ is said to be local if $K_{0}=K_{1}$. If $\phi$ is local we may have either $\phi\left(K_{0}\right)=\phi\left(K_{1}\right)=0$ (in which case $\phi$ is upper adjacent to $K_{0}$ ) or $\phi\left(K_{0}\right)=\phi\left(K_{1}\right)=1$ (in which case $\phi$ is lower adjacent to $K_{1}$ ). These two types and the atomic case in which $K_{0}<K_{1}$ are the only types which occur when $\mathscr{L}$ is a nest.

Definition. $\phi$ is said to be semi-local if $K_{1}<K_{0}$ and $\phi\left(K_{1}\right)=$ $\phi\left(K_{0}\right)$.

If $\phi$ is semi-local and the common value of $\phi$ is 0 then $\phi$ is upper adjacent to $K_{0}$; if the common value is 1 then $\phi$ is lower adjacent to $K_{1}$. While these two types cannot occur in nest algebras, they are reasonably similar to the nest algebra types.

Definition. $\phi$ is said to be diffuse if $K_{1}<K_{0}, \phi\left(K_{1}\right)=0$, and $\phi\left(K_{0}\right)=1$.

This is the one remaining type. The diffuse homomorphisms are the only ones which do not arise from a fundamental projection.

It might be tempting to suspect that $\cap\left\{\mathscr{A}_{\phi} \mid \phi\right.$ is not diffuse $\}$, rather than $\mathscr{F}$, is equal to the radical. That this is not so can be seen by considering the case in which $\mathscr{A}$ is a maximal abelian von Neumann algebra with no atoms. It is not difficult to see that in this case every lattice homomorphism on $\mathscr{L}=$ Lat $\mathscr{A}$ is diffuse.

3. A sufficient condition for an operator $A$ in $\mathscr{A}$ to belong to the radical of $\mathscr{A}$ is that $A \in \mathscr{F}$. In this section we give a necessary 
condition for $A$ to be in the radical. This condition will not, in general, be sufficient; the set of operators which satisfy this condition forms a closed, two-sided ideal which may properly contain $\mathscr{R}$.

DEFinition. If $E_{1}, E_{2}$ are nonzero orthogonal intervals from $\mathscr{L}$ such that $E_{1} \mathscr{B}(\mathscr{C}) E_{2} \subseteq \mathscr{L}$, we say $E_{1}, E_{2}$ is strictly ordered and write $E_{1} \ll E_{2}$

\section{LEMMA 14. The relation $\ll$ is transitive.}

Proof. Assume $E \ll F$ and $F \ll G$. We must show that $E \perp G$ and $E \mathscr{B}(\mathscr{H}) G \cong \mathscr{A}$. Let $P$ be the upper endpoint of $G$, viz the smallest projection in $\mathscr{L}$ which contains $G$. Then $G=P-N$, for some $N \in \mathscr{L}$ with $N \leqq P$. Since $F \ll G$, we have $F \leqq P$ and $F \perp G$, hence $F \leqq N$. Since $E \ll F$, we have that $E$ is contained in the upper endpoint for $F$, which is a subprojection of $N$. But the fact that $N \perp G$ now implies $E \perp G$.

Now suppose that $A \in E \mathscr{B}(\mathscr{C}) G$, so $A=E A G$. We must show that $A$ leaves invariant each member of $\mathscr{L}$. Fix $L \in \mathscr{L}$. If $G L=0$ then $A L=0$ and $A$ leaves $L$ invariant. Assume $G L \neq 0$ and let $x$ be a vector in $G L$. Let $y$ be an arbitrary vector in $E$ and let $z$ be any nonzero vector in $F$. By the assumption that $E \ll F$ and $F \ll G$, there exist operators $S, T \in \mathscr{A}$ such that $S z=y$ and $T x=z$. Thus $y=S T x$. Since $L$ is invariant under $\mathscr{A}$ and $x \in L$, we have $y \in L$ for all $y \in E$, i.e., $E \leqq L$. But $A=E A G$, hence $A L=L A L$ and $A \in \mathscr{A}$.

Definition. A mutually orthogonal family of intervals is said to be strictly ordered if it is linearly ordered by the relation $\ll$. The length of such a family is its cardinality.

Definition. If $A \in \mathscr{A}$ and $\varepsilon>0$ we define the $\varepsilon$-order of $A$ to be the number $R_{\varepsilon}(A)=\sup \{n \mid$ there exists a strictly ordered family $\mathscr{F}$ of length $n$ with $\|E A E\| \geqq \varepsilon$ for all $E \in \mathscr{F}\}$.

REMARK. It is clear that if $\varepsilon_{2}>\varepsilon_{1}>0$ then $R_{\varepsilon_{1}}(A) \geqq R_{\varepsilon_{2}}(A)$.

Lemma 15. If $A, B, C \in \mathscr{A}$ and $\varepsilon>0$, then $R_{\varepsilon}(A+B) \leqq R_{\varepsilon / 2}(A)+$ $R_{\varepsilon / 2}(B)$, and $R_{\varepsilon}(B A C) \leqq R_{\delta}(A)$, where $\delta=\varepsilon /\|B\|\|C\|$.

Proof. The first assertion follows from the observation that if $\|E(A+B) E\| \geqq \varepsilon$, then either $\|E A E\| \geqq \varepsilon / 2$ or $\|E B E\| \geqq \varepsilon / 2$. The second follows from the fact that for an interval $E$ we have $\|E(B A C) E\|=\|E B E A E C E\| \leqq\|B\|\|C\|\|E A E\|$, so that if $\|E(B A C) E\| \geqq$ $\varepsilon$, then $\|E A E\| \geqq \varepsilon /\|B\|\|C\|$. 
Definition. Let $\mathscr{J}=\left\{A \in \mathscr{A} \mid R_{\varepsilon}(A)<\infty\right.$ for every $\left.\varepsilon>0\right\}$.

LeMma 16. $\mathscr{J}$ is a closed 2-sided ideal in $\mathscr{A}$.

Proof. By Lemma 15 it is sufficient to show that $\mathscr{J}$ is closed. Suppose $A_{n} \in \mathscr{J}$ and $A_{n} \rightarrow A$. Fix $\varepsilon>0$ and choose $n$ so that $\left\|A-A_{n}\right\|<\varepsilon / 2$. If $E$ is an interval then $\|E A E\| \geqq \varepsilon$ implies $\left\|E A_{n} E\right\| \geqq$ $\left|\|E A E\|-\left\|E\left(A-A_{n}\right) E\right\|\right| \geqq \varepsilon / 2$. So $N_{\varepsilon}(A) \leqq N_{\varepsilon / 2}\left(A_{n}\right)<\infty$. Since $\varepsilon$ is arbitrary, $A \in \mathscr{F}$.

REMARK. In some cases, $\mathscr{J}$ may have nonzero intersection with the diagonal $\mathscr{A} \cap \mathscr{A}^{*}$. For example, if $E$ is an atom for the lattice $\mathscr{L}$ (for each projection $L \in \mathscr{L}$, either $L \geqq E$ or $L \perp E$ ) and if $A=$ $E A E$ then $R_{\varepsilon}(A) \leqq 1$ for every $\varepsilon$.

Lemma 17. Let $A \in \mathscr{A}$ and suppose that $A=L A L^{\perp}$, for some $L \in \mathscr{L}$. If $\left\{E_{1}, E_{2}\right\}$ is a strictly ordered pair of intervals then either $E_{1} A E_{1}=0$ or $E_{2} A E_{2}=0$.

Proof. We may suppose $E_{2} \ll E_{1}$, so that $E_{2} \mathscr{B}(\mathscr{H}) E_{1} \subseteq \mathscr{A}$. If $E_{1} A E_{1} \neq 0$ we have $E_{1} L \neq 0$. Let $x$ be a vector in $E_{1} L$. Since $E_{2} \ll$ $E_{1}, E_{2}$ is contained in any projection which is invariant under $\mathscr{A}$ and which contains $x$; in particular, $E_{2} \leqq L$. But then $E_{2} A E_{2}=0$.

Lemma 18. Let $A_{i} \in \mathscr{A}, L_{i} \in \mathscr{L}, i=1, \cdots, n$. Let $A=\sum_{i=1}^{n} L_{i} A L_{i}^{\perp}$. If $E_{1}, \cdots, E_{k}$ is a strictly ordered set with $E_{j} A E_{j} \neq 0$ for all $j$, then $k \leqq n$.

Proof. This follows from Lemma 17.

REMARK. Lemma 18 shows already that $\mathscr{J} \subseteq \mathscr{J}$. We shall prove below that $\mathscr{R} \cong \mathscr{J}$.

Lemma 19. Let $A \in \mathscr{A}$ and assume that $R_{\varepsilon}(A)=\infty$ for some $\varepsilon>0$. Let $E_{1}, \cdots, E_{n}$ be a finite set of mutually orthogonal simple projections with $\sum_{i=1}^{n} E_{i}=1$. Then $R_{\varepsilon}\left(E_{i} A E_{i}\right)=\infty$ for at least one $i$.

Proof. It suffices to prove the assertion for $n=2$. Suppose $E_{1}$ and $E_{2}$ are simple with $E_{1} \perp E_{2}, E_{1}+E_{2}=1$. Let $B=E_{1} A E_{1}+E_{2} A E_{2}$ and $C=E_{1} A E_{2}+E_{2} A E_{1}$. We claim first that $C$ can be expressed as a finite sum $C=\sum_{i=1}^{m} L_{i} C_{i} L_{i}$, for appropriate $C_{i} \in \mathscr{A}, L_{i} \in \mathscr{L}$. Indeed, $C$ is a sum of terms of the form $F A G$, where $F$ and $G$ are orthogonal intervals. If $F=M-N$ with $M>N, M, N \in \mathscr{L}$ then $F A G=$ $F A G F^{\perp}=M N^{\perp} A G\left(M^{\perp}+N\right)=M\left(N^{\perp} A G\right) M^{\perp}$. 
Let $k$ be an arbitrary positive integer and let $\left\{F_{j}\right\}$ be a strictly ordered family of length $k+m$ such that $\left\|F_{j} A F_{j}\right\| \geqq \varepsilon$, for all $j$. Since $A=B+C$, Lemma 18 and the transitivity of $\ll$ imply that there is a strictly ordered subfamily $\left\{F_{j}^{1}\right\}$ of length $k$ such that $\left\|F_{j}^{1} B F_{j}^{1}\right\| \geqq \varepsilon$ for all $j$. Since $\left\|F_{j}^{1} B F_{j}^{1}\right\|=\max _{i=1,2}\left\|F_{j}^{1} E_{i} A E_{i} F_{j}^{1}\right\|$ and $k$ is arbitrary, either $R_{\varepsilon}\left(E_{1} A E_{1}\right)=\infty$ or $R_{\varepsilon}\left(E_{2} A E_{2}\right)=\infty$.

Lemma 20. Let $A$ be an element of for which $R_{\varepsilon}(A)=\infty$, for some $\varepsilon>0$. Then there exist infinite sequences $E_{n}, K_{n}$ of intervals such that $E_{n} \perp K_{n}, E_{n+1}<K_{n}, K_{n+1}<K_{n}, R_{\varepsilon}\left(E_{n} A E_{n}\right) \geqq n$, and $R_{\varepsilon}\left(K_{n} A K_{n}\right)=\infty$, for all $n$.

Proof. Let $E_{11} \ll E_{12}$ be strictly ordered intervals with $\left\|E_{1 i} A E_{1 i}\right\| \geqq$ $\varepsilon$, for $i=1,2$. If $E_{11} A E_{11}$ has finite $\varepsilon$-order, let $E_{1}=E_{11}$ and let $K_{1}$ be an interval in $E_{1}^{\perp}$ such that $R_{\varepsilon}\left(K_{1} A K_{1}\right)=\infty$. If $E_{11} A E_{11}$ has infinite $\varepsilon$-order, let $E_{1}=E_{12}$ and $K_{1}=E_{11}$. In either case, $E_{1} \perp K_{1}, R_{\varepsilon}\left(E_{1} A E_{1}\right) \geqq$ 1 , and $R_{\varepsilon}\left(K_{1} A K_{1}\right)=\infty$.

Now assume inductively that intervals $E_{i}, K_{i}, i=1, \cdots, n$ have been constructed such that $E_{i+1}<K_{i}$ and $K_{i+1}<K_{i}$, for $i=1, \cdots$, $n-1$ and $E_{i} \perp K_{i}, R_{\varepsilon}\left(E_{i} A E_{i}\right) \geqq i, R_{\varepsilon}\left(K_{i} A K_{i}\right)=\infty$, for $i=1, \cdots, n$. Since $R_{\varepsilon}\left(K_{n} A K_{n}\right)=\infty$, there exist strictly ordered intervals $E_{n+1,1} \ll$ $E_{n+1,2} \ll \cdots \ll E_{n+1,2 n+2}$ contained in $K_{n}$ with $\left\|E_{n+1, i} A E_{n+1, i}\right\| \geqq \varepsilon$ for all $i$. Let $L$ be the upper endpoint of $E_{n+1, n+1}$ and let $F_{1}=L \wedge K_{n}$, $F_{2}=L^{\perp} \wedge K_{n}$. Then $F_{1}$ is an interval which contains each of $E_{n+1,1}$, $\cdots, E_{n+1, n+1}$ while $F_{2}$ is an interval containing $E_{n+1, n+2}, \cdots, E_{n+1,2 n+2}$. Therefore, $R_{\varepsilon}\left(F_{i} A F_{i}\right) \geqq n+1$, for $i=1$, 2. If $F_{1} A F_{1}$ has finite $\varepsilon$-order let $E_{n+1}=F_{1}$ and let $K_{n+1}$ be an interval contained in $K_{n}-E_{n+1}$ for which $R_{\varepsilon}\left(K_{n+1} A K_{n+1}\right)=\infty$. If $F_{1} A F_{1}$ has infinite $\varepsilon$-order, let $E_{n+1}=F_{2}$ and $K_{n+1}=F_{1}$. In either case $E_{n+1}$ and $K_{n+1}$ are orthogonal intervals, both contained in $K_{n}$, with $R_{\varepsilon}\left(E_{n+1} A E_{n+1}\right) \geqq n+1$ and $R_{\varepsilon}\left(K_{n+1} A K_{n+1}\right)=$ $\infty$. Induction completes the proof.

Lemma 21. Let $E_{1} \gg E_{2} \gg \cdots \gg E_{n}$ be, a strictly ordered set of intervals and let $A$ be an operator in $\mathscr{A}$ for which $\left\|E_{i} A E_{i}\right\|>1$, $i=1, \cdots, n$. Then there exists a contraction $S$ in $\mathscr{A}$ with support and range contained in $E=\sum E_{i}$ such that $S A$ is nilpotent of index $n$ and $\left\|E(S A)^{k} E\right\| \geqq 1$, for $k=1, \cdots, n-1$.

Proof. For each $i$, let $x_{i}$ be a unit vector in $E_{i}$ such that $\left\|E_{i} A E_{i} x_{i}\right\| \geqq 1$. Let $y_{i}=E_{i} A x_{i}$ and let $S=\sum_{i=1}^{n-1}\left\|y_{i}\right\|^{-2} y_{i} \otimes x_{i+1}$. (The operator $y \otimes x$ is defined by $y \otimes x(z)=\langle z, y\rangle x$, for all $z \in \mathscr{H}$.) Since the $E_{i}$ are mutually orthogonal, $\|S\|=\max _{i}\left\|y_{i}\right\|^{-2}\left\|y_{i} \otimes x_{i+1}\right\|=$ $\max _{i}\left\|y_{i}\right\|^{-1} \leqq 1$. The fact that the $E_{i}$ are strictly ordered guarantees that $S \in \mathscr{A}$. Observe also that $(S B)^{n}=0$, for all $B \in \mathscr{A}$. If $k=1, \cdots$, 
$n-1$, then $(S A)^{k} x_{1}=x_{k+1}+z$, for some vector $z$ orthogonal to $x_{k+1}$; hence $\left\|E(S A)^{k} E\right\| \geqq 1$. Thus $S$ satisfies the requirements of the lemma.

Proposition 22. If $A$ is in the radical of $\mathscr{A}$ then the e-order of $A$ is finite for all $\varepsilon>0$; i.e., $\mathscr{R} \subseteq \mathscr{J}$.

Proof. We must show that if $A \in \mathscr{A}$ and $R_{\varepsilon}(A)=\infty$ for some $\varepsilon>0$, then $A \notin \mathscr{R}$. We may assume $\varepsilon>1$ by multiplying by a scalar, if necessary. By Lemma 20 there exists a sequence $\left(E_{n}\right)$ of mutually orthogonal intervals such that $R_{\varepsilon}\left(E_{n} A E_{n}\right) \geqq n$, for all n. For each $n$ there exists a strictly ordered family $E_{n 1} \ll E_{n 2} \ll \cdots \ll E_{n n}$ contained in $E_{n}$ such that $\left\|E_{n i} A E_{n i}\right\| \geqq \varepsilon>1, i=1, \cdots, n$. By Lemma 21 there exists a contraction $S_{n}$ in $\mathscr{C}$ with $S_{n}=E_{n} S_{n} E_{n}$ such that $\left\|E_{n}\left(S_{n} A\right)^{n-1} E_{n}\right\|>1$. Let $S=\sum_{n=1}^{\infty} S_{n}$, the sum converging strongly. We have, for each $n \geqq 2$,

$$
\begin{aligned}
\left\|(S A)^{n-1}\right\| & \geqq\left\|E_{n}(S A)^{n-1} E_{n}\right\| \\
& =\left\|E_{n}\left(\left(E_{n} S E_{n}\right) A\right)^{n-1} E_{n}\right\| \\
& =\left\|E_{n}\left(S_{n} A\right)^{n-1} E_{n}\right\|>1 .
\end{aligned}
$$

Hence $S A$ is not quasinilpotent and $A \notin \mathscr{R}$.

4. If $\mathscr{A}$ is a nest algebra, then by a result of Lance [5], there is an isometric representation of each quotient algebra, $\mathscr{D}_{\phi}=\mathscr{A} / \mathscr{A}_{\phi}$, as an algebra of operators on a Hilbert space. Propositions 1 and 9 permit us to exhibit similar isometric representations of $\mathscr{D}_{\phi}$ and $\mathscr{D}=\mathscr{A} / \mathscr{F}$ as algebras of operators on a Hilbert space in the general case in which $\mathscr{A}$ is a reflexive operator algebra with commutative subspace lattice $\mathscr{L}$, acting on the Hilbert space $\mathscr{L}$.

THEOREM 23. For each $\phi \in X$, there is an isometric representation of $\mathscr{D}_{\phi}$ as an algebra of operators on Hilbert space.

Proof. If $\phi$ is atomic, let $E$ be the minimal projection in $\mathscr{F}_{\phi}$. It is easy to see that $\mathscr{D}_{\phi}$ is isometrically isomorphic to $\mathscr{B}(E \mathscr{H})$. (See [3], p. 381.) Assume henceforth that $\phi$ is not atomic. Let $B\left(\mathscr{F}_{\phi}\right)$ denote the set of all bounded, complex valued functions defined on $\mathscr{F}_{\phi}$. With the usual pointwise algebraic operations, complex conjugation as an involution, and the supremum norm, \|\|$_{\infty}, B\left(\mathscr{F}_{\phi}\right)$ forms an abelian unital $C^{*}$-algebra. If $f \in B\left(\mathscr{F}_{\phi}\right)$, let us say $\lim f=p$ provided that, for each $\varepsilon>0$ there is an element $E \in \mathscr{F}_{\phi}$, such that if $F \in \mathscr{F}_{\phi}$ and $F \leqq E$ then $|f(F)-p|<\varepsilon$. The set of all $f$ in $B\left(\mathscr{F}_{\phi}\right)$ for which $\lim f$ exists is a unital $C^{*}$-subalgebra of $B\left(\mathscr{F}_{\phi}\right)$; $\lim$ is a pure state on this sub-algebra. Let LIM be a pure state on $B\left(\mathscr{F}_{\phi}\right)$ which extends lim. 
LIM is a multiplicative, positive linear functional on $B\left(\mathscr{F}_{\phi}\right)$. Further, if $f(E) \geqq 0$ for all $E \leqq F$, for some fixed $F \in B\left(\mathscr{F}_{\phi}\right)$, then $\operatorname{LIM}(f) \geqq 0$. Indeed, if $f_{1}$ is defined by $f_{1}(E)=0$ when $E \leqq F$ and $f_{1}(E)=f(E)$ otherwise, then $\operatorname{LIM} f_{1}=\lim f_{1}=0$ and $\operatorname{LIM} f=\operatorname{LIM} f-$ $\operatorname{LIM} f_{1}=\operatorname{LIM}\left(f-f_{1}\right) \geqq 0$, since $f-f_{1} \geqq 0$. From this it follows that if $f, g \in B\left(\mathscr{F}_{\phi}\right)$ and $f(E) \leqq g(E)$, for all $E \leqq F$, for some fixed $F$, then LIM $f \leqq \operatorname{LIM} g$. For convenience, we frequently write $\operatorname{LIM}_{E} f(E)$ in place of $\operatorname{LIM} f$.

Let $B\left(\mathscr{F}_{\phi}, \mathscr{C}\right)$ be the linear space of all bounded functions on $\mathscr{F}_{\phi}$ with values in $\mathscr{H}$. For all $x, y \in B\left(\mathscr{F}_{\phi}, \mathscr{H}\right)$, the function $E \rightarrow$ $\langle E x(E), y(E)\rangle$ is in $B\left(\mathscr{F}_{\phi}\right)$, hence we can define a sesquilinear form on $B(\mathscr{F}, \mathscr{H})$ by

$$
\langle x, y\rangle=\operatorname{LIM}_{E}\langle E x(E), y(E)\rangle .
$$

Let. $\mathscr{N}=\left\{x \in B\left(\mathscr{F}_{\phi}, \mathscr{C}\right) \mid \mathrm{LIM}_{E}\|E x(E)\|=0\right\} . \mathscr{N}$ is a linear subspace of $B\left(\mathscr{F}_{\phi}, \mathscr{K}\right)$; let $\mathscr{K}^{1}$ be the quotient space. Observe that if $x \in \mathscr{N}$ and $y \in B\left(\mathscr{F}_{\phi}, \mathscr{C}\right)$ then $\langle x, y\rangle=0$. Indeed, $|\langle x, y\rangle| \leqq\langle x, x\rangle^{1 / 2}\langle y, y\rangle^{1 / 2}$ and $\langle x, x\rangle=\operatorname{LIM}_{E}\langle E x(E), x(E)\rangle=\operatorname{LIM}_{E}\|E x(E)\|^{2}=0$. This implies that the sesquilinear form on $B\left(\mathscr{F}_{\dot{\phi}}, \mathscr{C}\right)$ induces an inner product on the quotient space $\mathscr{K}^{1}$. For any $x \in B\left(\mathscr{F}_{\phi}, \mathscr{H}\right)$, let $\bar{x}$ denote the image of $x$ in the quotient $\mathscr{K}^{-1}$. The inner product on $\mathscr{K}^{-1}$ is given by $\langle\bar{x}, \bar{y}\rangle=\operatorname{LIM}_{E}\langle E x(E), y(E)\rangle$. Let $\mathscr{K}$ be the completion of $\mathscr{K}^{1}$; $\mathscr{K}$ is the Hilbert space on which we construct a representation of $\mathscr{D}_{\dot{\phi}}$.

For each operator $A$ in $\mathscr{A}$ define a linear mapping $\Pi_{0}(A)$ on $B\left(\mathscr{F}_{\phi}, \mathscr{C}\right)$ by $\left(\Pi_{0}(A) x\right)(E)=E A E x(E)$, for all $E \in \mathscr{F}_{\phi}$. Observe first that $\Pi_{0}(A)$ leaves $\mathscr{N}$ invariant. Let $x \in \mathscr{N}$. Then

$$
\begin{aligned}
0 & \leqq \operatorname{LIM}_{E}\left\|E \Pi_{0}(A) x(E)\right\|=\operatorname{LIM}_{E}\|E A E x(E)\| \leqq\|A\| \operatorname{LIM}_{E}\|E x(E)\| \\
& =0
\end{aligned}
$$

hence $\Pi_{0}(A) x \in \mathscr{N}$. From this it follows that $\Pi_{0}(A)$ induces an operator $\Pi(A)$ acting on the quotient space $\mathscr{K}^{1}$. Note that $\Pi(A)$ is determined by the condition

$$
\langle\Pi(A) \bar{x}, \bar{y}\rangle=\operatorname{LIM}_{E}\langle E A E x(E), y(E)\rangle .
$$

Since $\Pi_{0}$ is an algebra homomorphism on $\mathscr{A}$, so is $I I$. (This uses the fact that for any interval $E$, the mapping $A \rightarrow E A E$ is multiplicative on $\mathscr{A}$.)

We now compute the norm of $\Pi(A)$ as an operator on the preHilbert space $\mathscr{K}^{1}$. Let $\bar{x}$ and $\bar{y}$ be unit vectors in $\mathscr{K}^{1}$. Let $F$ be an arbitrary interval in $\mathscr{F}_{\phi}$. Then, for any $E \in \mathscr{F}_{\phi}$ with $E \leqq F$, we have $\|E A E\| \leqq\|F A F\|$, and hence 


$$
|\langle E A E x(E), y(E)\rangle| \leqq\|F A F\|\|E x(E)\|\|E y(E)\| \text { • }
$$

Consequently,

$$
\begin{aligned}
|\langle\Pi(A) \bar{x}, \bar{y}\rangle| & =\left|\operatorname{LIM}_{E}\langle E A E x(E), y(E)\rangle\right| \\
& \leqq \operatorname{LIM}_{E}|\langle E A E x(E), y(E)\rangle| \\
& \leqq\|F A F\| \operatorname{LIM}_{E}\|E x(E)\| \operatorname{LIM}_{E}\|E y(E)\| \\
& \leqq\|F A F\| .
\end{aligned}
$$

Since $F$ is arbitrary, $\|\Pi(A)\| \leqq \inf \left\{\|F A F\| \mid F \in \mathscr{F}_{\phi}\right\}=N_{\phi}(A)$.

In fact, we actually have equality. Let $\varepsilon>0$ be arbitrary. For each $E \in \mathscr{F}_{\phi}$, there is a vector $x(E) \in E \mathscr{H}$ such that $\|x(E)\|=1$ and $\|E A E x(E)\| \geqq N_{\dot{\phi}}(A)-\varepsilon$. Then,

$$
\begin{aligned}
\|\Pi(A) \bar{x}\|^{2} & =\langle\Pi(A) \bar{x}, \Pi(A) \bar{x}\rangle \\
& =\operatorname{LIM}_{E}\langle E A E x(E), E A E x(E)\rangle \\
& =\operatorname{LIM}_{E}\|E A E x(E)\|^{2} \\
& \geqq\left(N_{\phi}(A)-\varepsilon\right)^{2} .
\end{aligned}
$$

Since $\|\bar{x}\|=1$, we have $\|\Pi(A)\| \geqq N_{\phi}(A)-\varepsilon$, and since $\varepsilon$ is arbitrary, $\|\Pi(A)\|=N_{\phi}(A)$.

For each $A \in \mathscr{A}, \Pi(A)$ has a unique extension to a bounded linear operator, which we also denote by $\Pi(A)$, acting on the Hilbert space $\mathscr{K}$. Thus $\Pi$ is a representation of $\mathscr{A}$ acting on $\mathscr{K}$ for which $\|\Pi(A)\|=N_{\phi}(A)$, for all $A \in \mathscr{A}$. From Proposition 1 it is clear that $\Pi$ induces an isometric representation of $\mathscr{D}_{\phi}$ acting on $\mathscr{K}$.

CoROLlary 24. There is an isometric representation of $\mathscr{D}=$ $\mathscr{F} / \mathscr{I}$ as an algebra of operators acting on Hilbert space.

Proof. For each $\phi \in X$, let $\Pi_{\phi}$ be an isometric representation of $\mathscr{D}_{\phi}$ acting on a Hilbert space $\mathscr{H}_{\phi}$. Let $q_{\phi}: \mathscr{A} \rightarrow \mathscr{D}_{\phi}$ and $q: \mathscr{A} \rightarrow \mathscr{D}$ be the canonical quotient maps. Let $\mathscr{H}=\sum_{\phi \in X}^{\oplus} \mathscr{H}_{\phi}$. Define $\Pi_{0}: \mathscr{A} \rightarrow$ $\mathscr{L}(\mathscr{H})$ by $\Pi_{0}(A)=\sum_{\phi \in X}^{\oplus} \Pi_{\phi}\left(q_{\phi}(A)\right)$. Then $\Pi_{0}$ is a representation of A2. For each $A \in \mathscr{A},\left\|\Pi_{0}(A)\right\|=\sup _{\phi}\left\|\Pi_{\phi} q_{\phi}(A)\right\|=\sup _{\phi} N_{\phi}(A)=\|q(A)\|$, by Proposition 9 . Hence $\Pi_{0}$ induces an isometric representation, $\Pi$, of $\mathscr{D}$ acting on $\mathscr{H}$.

5. Let $\mathscr{A}$ be a reflexive operator algebra with commutative subspace lattice $\mathscr{L}$. Recall that the diagonal of $\mathscr{A}$ is defined to be the von Neumann algebra $\mathscr{A} \cap \mathscr{A}^{*}$ and the core of $\mathscr{A}$ is defined to be the von Neumann algebra generated by $\mathscr{L}$. We shall denote the diagonal and core of $\mathscr{A}$ by $\mathscr{A}_{d}$ and $\mathscr{A}_{c}$ respectively. Observe that $\mathscr{A}_{d}=\mathscr{A}_{c}^{\prime}$, 
i.e., the diagonal is the commutant of the core. As before, $\mathscr{J}=$ $\bigcap_{\phi \in X} \mathscr{X}_{\phi}$.

Definition. We define the $\mathscr{J}$-commutant of the core to be the algebra

$$
\mathscr{A}=\left\{A \in \mathscr{A} \mid A B-B A \in \mathscr{F}, \text { for all } B \in \mathscr{A}_{c}\right\} \text {. }
$$

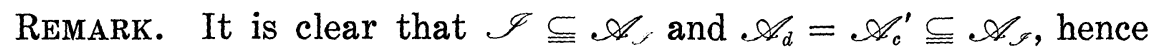
$\mathscr{A}_{d}+\mathscr{I} \subseteq \mathscr{A}_{\mathscr{S}}$. Since $\mathscr{A}_{d}$ is a $C^{*}$-algebra, it is semi-simple; since $\mathscr{I} \subseteq \mathscr{R}$, it follows that $\mathscr{I} \cap \mathscr{A}_{d}=(0)$, so $\mathscr{A}_{d}+\mathscr{I}=\mathscr{A}_{d} \oplus \mathscr{F}$. It is proven in [6] (Theorem 2.4) that if $\mathscr{L}$ is a nest then $\mathscr{A}_{d} \oplus \mathscr{I}=$ $\mathscr{A}_{\mathcal{S}}$. We prove below that the same result holds for commutative subspace lattices which satisfy certain additional hypotheses. It seems quite possible that the result holds in the general case, but we have not been able to prove it.

We sketch briefly some of the tools needed for the theorem. Full details, proofs, and/or references can be found in [6]. Let $M$ be an invariant mean on the (abelian) group, $\mathscr{C}$, of unitary operators in $\mathscr{A}_{c}$. If $g$ is a bounded, complex valued function on $\mathscr{C}$ we frequently write $M_{U} g(U)$ in place of $M g$. Let $\mathscr{C}_{1}$ denote the ideal of trace class operators in $B(\mathscr{C})$ and identify $B(\mathscr{H})$ as the dual of $\mathscr{C}_{1}$ via the pairing $(T, f)=\operatorname{Tr}(T f)$, for $T \in B(\mathscr{K}), f \in \mathscr{C}_{1}$. Define a mapping $\psi$ on $B(\mathscr{H})$ by the formula $(\psi(T), f)=M_{U}\left(U^{*} T U, f\right)$, for $T \in \mathscr{B}(\mathscr{H})$, $f \in \mathscr{C}_{1}$. The translation invariance of $M$ implies that $\psi$ maps $B(\mathscr{H})$ into $\mathscr{A}_{c}^{\prime}=\mathscr{A}_{d}$; one can verify that $\psi$ is a norm 1 projection of $\mathscr{B}(\mathscr{H})$ onto $\mathscr{A}_{d}$ which satisfies $\psi(A B)=A \psi(B), \psi(B A)=\psi(B) A$, for all $A \in \mathscr{A}_{d}, B \in \mathscr{B}(\mathscr{H})$. If $T \in \mathscr{B}(\mathscr{H})$, define $\delta T: \mathscr{A}_{c} \rightarrow B(\mathscr{H})$ by $\delta T(A)=A T-T A$. If $D: \mathscr{A}_{c} \rightarrow B(\mathscr{H})$ is any derivation (i.e., $D$ is linear and $D(A B)=A D(B)+D(A) B$, for all $\left.A, B \in \mathscr{A}_{c}\right)$ then $D=\delta T$, where $T$ is defined by $(T, f)=M_{U}\left(U^{*} D(U), f\right), f \in \mathscr{C}_{1}$. If $D=\delta T_{1}$, for some other $T_{1} \in \mathscr{B}(\mathscr{C})$, then $T=T_{1}-\psi\left(T_{1}\right)$.

Each of the following two hypotheses is satisfied by every nest on a separable Hilbert space and by some, but not all, nontotally ordered commutative subspace lattices. (Hypothesis A is actually satisfied by every nest regardless of the dimension of the Hilbert space.)

Hypothesis $A$. If $\left(F_{n}\right)$ is an infinite sequence of mutually orthogonal intervals from $\mathscr{L}$. then there exists an element $\psi$ in $X$ such that if $E \in \mathscr{F}_{\psi}$ then $F_{n} \leqq E$, for some $n$.

Hypothesis $B$. For each $\phi \in X$, there is a countable family $\left\{F_{n}\right\}$ 
of intervals in $\mathscr{F}_{\phi}$, totally ordered by inclusion, such that, for each $E \in \mathscr{F}_{\phi}, F_{n} \leqq E$, for some $n$.

THEOREM 25. If $\mathscr{A}$ satisfies either Hypothesis $A$ or Hypothesis $B$ then the $\mathscr{F}$-commutant of the core of $\mathscr{A}$ is equal to the direct sum of the diagonal of $\mathscr{A}$ and $\mathscr{I}$; i.e., $\mathscr{H}_{\mathcal{S}}=\mathscr{A}_{d} \oplus \mathscr{F}$.

Proof. From the remark at the beginning of this section, it suffices to prove that if $B \in \mathscr{A}$ and if $A B-B A \in \mathscr{F}$, for all $A \in \mathscr{A}_{c}$ then $B \in \mathscr{A}_{d}+\mathscr{F}_{\text {. }}$ For this, it suffices to show that $B-\psi(B) \in \mathscr{F}_{\text {. }}$ Let $T=B-\psi(B)$ and let $D=\delta B=\delta T$. If $T \notin \mathscr{F}$, then there exists an element $\phi \in X$ such that $N_{\phi}(T)>0$. Note that $\phi$ cannot be atomic, for otherwise $\mathscr{F}_{\phi}$ has a minimal projection $E_{0}$, for which $E_{0} T E_{0} \in \mathscr{A}_{d}$. But then $E_{0} T E_{0}=\psi\left(E_{0} T E_{0}\right)=E_{0} \psi(T) E_{0}=0$ and $N_{\phi}(T)=0$, a contradiction. Thus $\mathscr{F}_{\phi}$ is a directed family of projections whose strong limit is 0 .

Let $E \in \mathscr{F}_{\phi}$. The lower semi-continuity of norm and the fact that $\|E T E\| \geqq N_{\phi}(T)>(1 / 2) N_{\phi}(T)$ allows us to find a projection $E^{\prime} \leqq E, E^{\prime} \in$ $\mathscr{F}_{\phi}$ such that $\left\|\left(E-E^{\prime}\right) T\left(E-E^{\prime}\right)\right\|>(1 / 2) N_{\phi}(T)$. Since $E-E^{\prime}$ is a simple projection, it contains an interval $F$ for which $\|F T F\|>$ $(1 / 2) N_{\phi}(T)$. By repeating this argument, we may obtain inductively a mutually orthogonal sequence of intervals $\left(F_{n}\right)$ such that $\left\|F_{n} T F_{n}\right\|>$ $(1 / 2) N_{\phi}(T)$, all $n$. If Hypothesis $\mathrm{B}$ is satisfied we can also arrange to choose the $\left(F_{n}\right)$ so that any projection $E$ in $\mathscr{F}_{\phi}$ contains some $F_{n}$. If Hypothesis A is satisfied then there is an element $\psi$ in $X$ such that every projection in $\mathscr{F}_{\psi}$ contains some $F_{n}$.

We next claim that for each $n$, there is an operator $A_{n} \in \mathscr{A}_{c}$ such that $\left\|A_{n}\right\|=1$ and $\left\|F_{n} D\left(A_{n}\right) F_{n}\right\|>(1 / 2) N_{\phi}(T)$. Suppose the contrary, namely that there is an integer $n$ such that $\left\|F_{n} D(A) F_{n}\right\| \leqq$ $(1 / 2) N_{\phi}(T)$, for all $A \in \mathscr{A}_{c}$ with $\|A\| \leqq 1$. Then for any unitary operator $U$ in $\mathscr{A}_{c}$ we have

$$
\begin{aligned}
\left|\left(U^{*} F_{n} D(U) F_{n}, f\right)\right| & \leqq\|f\|_{1}\left\|U^{*} F_{n} D(U) F_{n}\right\| \\
& \leqq\|f\|_{1}\left\|F_{n} D(U) F_{n}\right\| \\
& \leqq \frac{1}{2} N_{\phi}(T)\|f\|_{1} \text { for all } f \in \mathscr{C}_{1} .
\end{aligned}
$$

Since $\left(F_{n} T F_{n}, f\right)=M_{U}\left(F_{n} U^{*} D(U) F_{n}, f\right)=M_{U}\left(U^{*} F_{n} D(U) F_{n}, f\right)$, we obtain $\left|\left(F_{n} T F_{n}, f\right)\right| \leqq(1 / 2) N_{\phi}(T)\|f\|_{1}$, for all $f \in \mathscr{C}_{1}$. This implies that $\left\|F_{n} T F_{n}\right\| \leqq(1 / 2) N_{\phi}(T)$, a contradiction. Thus the claim is established.

Since $A_{n} \in \mathscr{A}_{c}, A_{n} F_{n}=F_{n} A_{n}$ for all $n$. The fact that the $\left(F_{n}\right)$ are mutually orthogonal implies that $\sum_{n} A_{n} F_{n}$ converges strongly in $\mathscr{A}_{c}$; let $A=\sum_{n} A_{n} F_{n}$. Observe that $F_{n} D(A) F_{n}=F_{n}(A T-T A) F_{n}=$ 
$F_{n}\left(A_{n} T-T A_{n}\right) F_{n}=F_{n} D\left(A_{n}\right) F_{n}$, for all $n$. Hence $\left\|F_{n} D(A) F_{n}\right\| \geqq$ $(1 / 2) N_{\dot{\phi}}(T)$. For any projection $E$ in $\mathscr{F}_{\psi}$ (if Hypothesis A is satisfied) or $\mathscr{F}_{\phi}$ (if Hypothesis $\mathrm{B}$ is satisfied), we must therefore have $\|E D(A) E\| \geqq(1 / 2) N_{\phi}(T)$. Since $N_{\phi}(T)>0$, this implies that $D(A) \notin$ $\mathscr{F}$. But $D(A)=A B-B A \in \mathscr{F}$ by hypothesis, a contradiction. Thus we must have $T \in \mathscr{J}$ and the theorem is proven.

\section{REFERENCES}

1. W. Arveson, Operator algebras and invariant subspaces, Ann. of Math., 100 (1974), 433-532.

2. G. Birkhoff, Lattice Theory, 3rd ed. Amer. Math. Soc. Colloquium Publications, 25, Providence, R. I.

3. A. Hopenwasser, The radical of a reflexive operator algebra, Pacific J. Math., 65 (1976), 375-392.

4. J. Kelley, General Topology, D. Van Nostrand Company, New York.

5. E. C. Lance, Some properties of nest algebras, Proc. London Math. Soc., (3), 19 (1969), 45-68.

6. D. Larson, On the structure of certain reflexive operator algebras, To appear, J. Functional Anal.

7. J. Ringrose, On some algebras of operators, Proc. London Math. Soc., (3), 15 (1965), 61-83.

Received September 23, 1977. The first author was supported in part by a grant from the Research Grants Committee of the University of Alabama and by a grant from the N.S.F. The second author was supported in part by a grant from the N.S.F.

The University of Alabama

UNIVERSITY, AL 35476

AND

UNIVERSITY OF NEBRASKA

LINCOLN, NE 68588 


\title{
PACIFIC JOURNAL OF MATHEMATICS
}

\section{EDITORS}

\author{
DONALD BABBITT (Managing Editor) \\ University of California \\ Los Angeles, CA 90024 \\ HUGo ROSSI \\ University of Utah \\ Salt Lake City, UT 84112 \\ C. C. MOORE \\ University of California \\ Berkeley, CA 94720
}

J. DugundJI

Department of Mathematics

University of Southern California

Los Angeles, CA 90007

R. FinN and J. Milgram

Stanford University

Stanford, CA 94305

\section{ASSOCIATE EDITORS}
E. F. BECKENBACH
B. H. Neumann
F. WolF
K. YOSHIDA

\section{SUPPORTING INSTITUTIONS}

\author{
UNIVERSITY OF BRITISH COLUMBIA \\ CALIFORNIA INSTITUTE OF TECHNOLOGY \\ UNIVERSITY OF CALIFORNIA \\ MONTANA STATE UNIVERSITY \\ UNIVERSITY OF NEVADA, RENO \\ NEW MEXICO STATE UNIVERSITY \\ OREGON STATE UNIVERSITY \\ UNIVERSITY OF OREGON
}

UNIVERSITY OF SOUTHERN CALIFORNIA

STANFORD UNIVERSITY

UNIVERSITY OF HAWAII

UNIVERSITY OF TOKYO

UNIVERSITY OF UTAH

WASHINGTON STATE UNIVERSITY

UNIVERSITY OF WASHINGTON

The Supporting Institutions listed above contribute to the cost of publication of this Journal, but they are not owners or publishers and have no responsibility for its content or policies.

Mathematical papers intended for publication in the Pacific Journal of Mathematics should be in typed form or offset-reproduced, (not dittoed), double spaced with large margins. Please do not use built up fractions in the text of the manuscript. However, you may use them in the displayed equations. Underline Greek letters in red, German in green, and script in blue. The first paragraph or two must be capable of being used separately as a synopsis of the entire paper. Please propose a heading for the odd numbered pages of less than 35 characters. Manuscripts, in triplicate, may be sent to any one of the editors. Please classify according to the scheme of Math. Reviews, Index to Vol. 39. Supply name and address of author to whom proofs should be sent. All other communications should be addressed to the managing editor, or Elaine Barth, University of California, Los Angeles, California, 90024.

50 reprints to each author are provided free for each article, only if page charges have been substantially paid. Additional copies may be obtained at cost in multiples of 50 .

The Pacific Journal of Mathematics is issued monthly as of January 1966. Regular subscription rate: $\$ 72.00$ a year (6 Vols., 12 issues). Special rate: $\$ 36.00$ a year to individual members of supporting institutions.

Subscriptions, orders for numbers issued in the last three calendar years, and changes of address should be sent to Pacific Journal of Mathematics, P.O. Box 969, Carmel Valley, CA 93924, U.S.A. Older back numbers obtainable from Kraus Periodicals Co., Route 100, Millwood, NY 10546.

PUBLISHED BY PACIFIC JOURNAL OF MATHEMATICS, A NON-PROFIT CORPORATION

Printed at Kokusai Bunken Insatsusha (International Academic Printing Co., Ltd.). 8-8, 3-chome, Takadanobaba, Shinjuku-ku, Tokyo 160, Japan.

Copyright (C) 1979 by Pacific Journal of Mathematics Manufactured and first issued in Japan 


\section{Pacific Journal of Mathematics}

\section{Vol. 81, No. $2 \quad$ December, 1979}

Ersan Akyildiz, Vector fields and equivariant bundles ............... 283

Ehrhard Behrends, The centralizer of tensor products of Banach spaces ( $a$ function space representation) ......................... 291

Geoffrey R. Burton, Congruent sections of a convex body ............. 303

John Warnock Carlson, $H$-closed and countably compact extensions ...... 317

Robert Charles Carlson, Eigenfunction expansions for selfadjoint

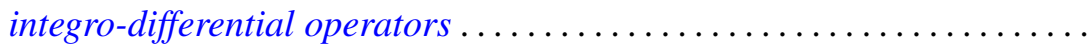

Robert Damiano, Coflat rings and modules.

Eric Karel van Douwen and Washek (Vaclav) Frantisek Pfeffer, Some properties of the Sorgenfrey line and related spaces ................

Uri Elias, Necessary conditions and sufficient conditions for disfocality and

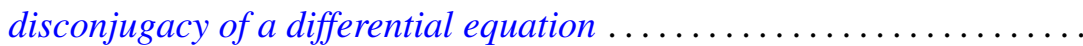

V. L. (Vagn Lundsgaard) Hansen, Polynomial covering spaces and homomorphisms into the braid groups .......................

Paul Hess, Dedekind's problem: monotone Boolean functions on the lattice

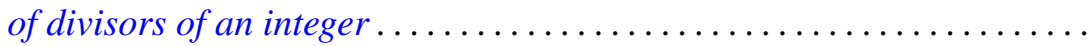

Alan Hopenwasser and David Royal Larson, The carrier space of a reflexive

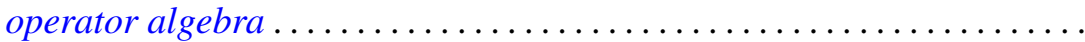

Kyung Bai Lee, Spaces in which compacta are uniformly regular $G_{\delta} \ldots \ldots$.

Claude Levesque, A class of fundamental units and some classes of

Jacobi-Perron algorithms in pure cubic fields...

Teck Cheong Lim, A constructive proof of the infinite version of the

Belluce-Kirk theorem ..........................

Dorothy Maharam and A. H. Stone, Borel boxes

Roger McCann, Asymptotically stable dynamical systems are linear

Peter A. McCoy, Approximation and harmonic continuation of axially symmetric potentials in $E^{3} \ldots \ldots \ldots \ldots \ldots \ldots \ldots$

Takahiko Nakazi, Extended weak-* Dirichlet algebras ....

Carl L. Prather, On the zeros of derivatives of balanced trigonometric polynomials ................................

Iain Raeburn, An implicit function theorem in Banach spaces...

Louis Jackson Ratliff, Jr., Two theorems on the prime divisors of zeros in completions of local domains...

Gloria Jean Tashjian, Cartesian-closed coreflective subcategories of

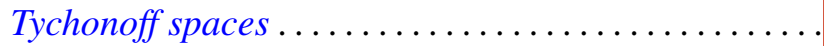

Stephen Edwin Wilson, Operators over regular maps.... . . 